Proceedings of the 2009 Winter Simulation Conference

M. D. Rossetti, R. R. Hill, B. Johansson, A. Dunkin, and R. G. Ingalls, eds.

\title{
EVALUATION OF MODELING, SIMULATION AND OPTIMIZATION APPROACHES FOR WORK FLOW MANAGEMENT IN SEMICONDUCTOR MANUFACTURING
}

\author{
Robert Kohn \\ Daniel Noack \\ Marcin Mosinski \\ Zhugen Zhou \\ Oliver Rose \\ Institute of Applied Computer Science \\ Dresden University of Technology \\ Dresden, 01062, GERMANY
}

\begin{abstract}
Work flow management is one of the key elements that bias the economic competitive position of a fabrication facility, especially in semiconductor manufacturing. This paper presents the goals, methods and challenges of our collaborative project with partners in semiconductor industry. The project activities concern topics in the disciplines of modeling, simulation and optimization classed among work flow management. Our goal is to evaluate suitable methods in the area of modeling, simulation and optimization in use cases given by reality. This project considers future performance prediction using different simulation techniques. In addition several optimization approaches will be evaluated in this project.
\end{abstract}

\section{INTRODUCTION}

Manufacturing industry steadily intends to increase their fabrication process productivity. Work flow management (WFM) is one of the key elements that bias the economic competitive position of a fabrication facility, especially in semiconductor manufacturing. Modeling, simulation and optimization of work flows are absolutely essential disciplines in that application area.

The complexity and highly dynamic behaviour of a wafer fabrication facility (waferfab) respectable complicates the process of modeling. Reliable models based on validated data state the groundwork of simulation and optimization applications. In particular the validation and verification of manufacturing data describes a stand alone topic. Automated model generation of tool(groups) and work flows is desirable, but hard to implement.

Simulation and optimization highly depend on defined use cases and their constraints. Detailed simulating and optimizing waferfab work flows easily exceeds current computational power due to high computational complexity. In consequence suitable subproblems and a acceptable level of detail have to be identified.

Simulation as an element in decision support systems got more and more popular with time. In particular the availability of future performance forecasts for short terms supports the process of decisioning. Forecasts considering the current waferfab state help for example to identify forthcoming bottlenecks. Such informations make interventions possible to prevent negative effects in manufacturing. Framed by this project discrete event simulation (DES) and alternative methods will be used to predict future performance.

Apart from simulation, optimization takes important place in WFM strategies. Optimization based on simulation, solver, heuristics or artificial intelligence techniques promises cost savings in manufacturing. As with simulation computational complexity is one of the most important criteria in evaluation process. Rule based dispatching is the state of the art at that time. There is a obvious tendency to simulation supported dispatching systems in semiconductor industry. Several manufacturers try to establish resource scheduling on operative level, so we. In fact scheduling on operative level is very hard to achieve in cause of high complexity. That means complexity of real manufacturing system as well as computational complexity of optimizing problems.

Based on a adequate model the usability of suitable approaches in reality scenarios will be explored. The estimated benefit in contrast to the degree of complexity determines the usability of dedicated methods in defined situations. This relation affects finally the acceptance of these methods in practice. 


\section{PROJECT OUTLINES}

The project activities concern topics in the disciplines of modeling, simulation and optimization in the field of frontend wafer fabrication. Our goal is to evaluate suitable methods in the area simulation and optimization in different use cases. This includes the examination of modeling approaches and their usability. In addition we assess the effort to realize benefits in practice. In cooperation with our industrial partners we try to generate valuable results under consideration of all constraints of a real world environment.

The project activities are structured as follows. Our project road map includes four key phases to achieve the desired milestones. Following four phases describe our main tasks we have to work on:

1. Analysis of systems and constraints.

2. Development of interfaces to manufacturing data.

3. Data validation and system model generation.

4. Evaluation of simulation and optimization approaches.

After analyzing systems and important constraints we start developing our data model. The development of a proper interface to manufacturing data results in an independent database. To model systems and work flows the validation of all generated data is essential. Based on appropriate data and system models we evaluate simulation and optimization approaches.

Our course of actions does not strictly follow the above mentioned project steps sequentially. Actually we iterate these four phases in cyclic manner. That cyclic aspect enables us to consider early insights we achieve in different project phases. We focus on use case cutouts to gain experiences in modeling, simulation and optimization. If necessary we modify our models to ensure results of high quality.

There are following main work packages identified in the mentioned project:

- Modeling equipments and work flows with respect to different levels of details.

- Simulation of work flows for short term horizon using discrete event simulation (DES).

- Future performance prediction using alternative methods.

- Optimization of work flows in different application areas evaluating different approaches.

Modeling equipment(groups) and work flows in a adequate way builds the groundwork of our project. Data collection including following validation and verification of these takes much proportion of our project resources. The question of a sufficient level of detail in contrast to acceptable complexity will be examined. Our project relates directly to current research projects about fabrication modeling methodologies. We try to merge industrial constraints with academic research on tool independent description languages.

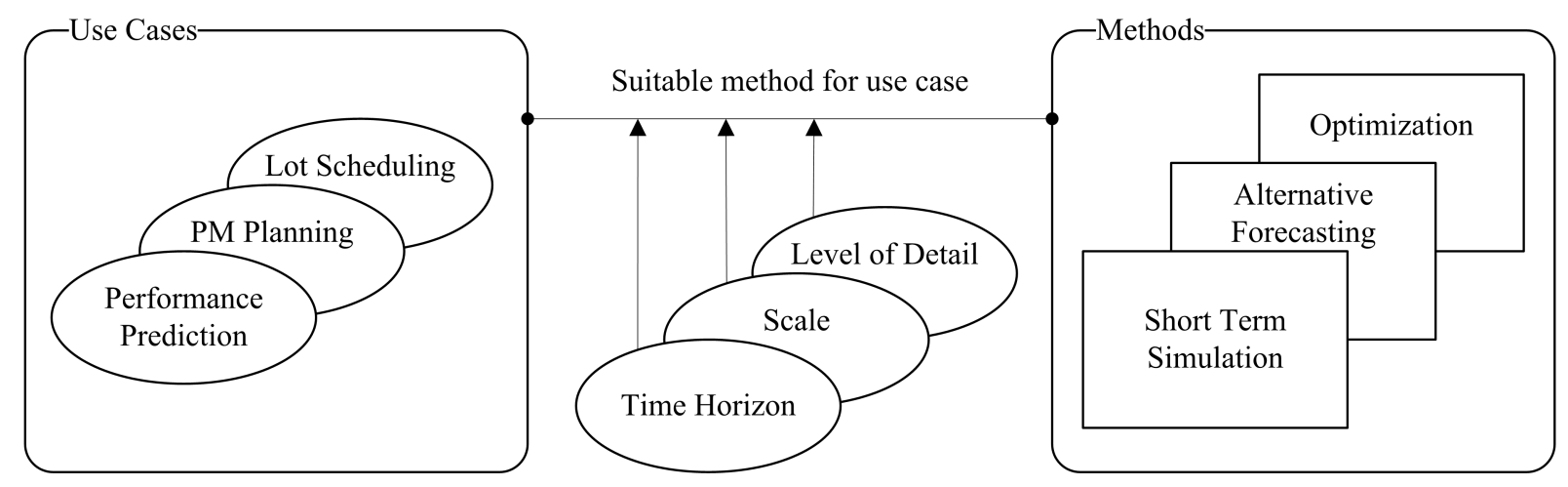

Figure 1: Scope of our project

With time simulation became an essential part in the management of work flows. In particular discrete event simulation for short terms represents an important part in our project concept. Short term simulation aims on prediction of waferfab future performance, e.g. detection of forthcoming bottlenecks. At least simulation provides the capability to valuate several waferfab scenarios and support WFM decisioning. 


\section{Kohn, Noack, Mosinski, Zhou, and Rose}

Alternative Forecasting is competitively used to discrete event simulation in our project. Evaluating alternative methods to predict waferfab future performance is the objective of this project part. Later on DES and alternative methods for forecasts will be compared on their performance characteristics.

In fact our project is actually aimed on optimization of work flows to realize cost saving potentials. We focus on simulation based optimization, that is characterized by cyclic simulation runs in combination with derived decisions. Moreover we deal with solver based optimization, heuristics and techniques originated from the scope of artificial intelligence.

The mentioned work packages mostly match in their needs with respect to acquisition of manufacturing data. We expect synergy effects in using the same data models that are adjustable for work package specific requirements. In addition the cross usage of results between work packages seems to be desirable. Maybe simulation provides future lot arrivals to feed optimization approaches. All of these work packages in the scope of modeling, simulation and optimization are basically determined by identical requirements. Following requirements that influence each other mainly result in the degree of computational complexity:

- Level of detail of models that affect directly simulation and optimization approaches.

- Scale of models that ranges between single equipment operations and full fabrication facility.

- Time horizon of simulation runs and optimization problems.

Under consideration of these requirements we evaluate methods in the area of modeling, simulation and optimization. We need to know, which method is suitable in which use case and what is needful to realize an estimated benefit. Considerable use cases are listed below.

- On line scheduling on lot level.

- Preventive maintenance (PM) planning.

- Evaluation of impacts caused by equipment dedicated processes.

- Assessment of dispatch and equipment setup changing strategies.

- Detailed future performance prediction for short terms.

\section{MODELING AND INFORMATION QUALITY}

This section gives a short insight into challenges of modeling in today's semiconductor industry. Modeling the focused fabrication system enables the evaluation of simulation and optimization techniques. Reliable models of systems include reliable data models based on real manufacturing data. Modeling takes a lot of project resources and influences extraordinary the quality of our results.

Models of manufacturing environments mostly depend on the concrete implementations in a concrete modeling software. The usage of software independent modeling approaches to describe equipments and work flows is desirable. Modeling in standardized modeling languages like SysML seems to be very helpful in this context (Schönherr 2008). Current research projects try to use knowledge and standards in the field of software development for modeling manufacturing systems. Especially the modeling of semiconductor tools requires fundamental research activities in this application area.

With respect to queueing theory a semiconductor fabrication facility is mainly characterized by the structure of job shop floors. Here, a huge variety of equipments exists, for example complex cluster equipments. In addition the use of complex technologies within the fabrication process offers more challenges like:

- Dedications for processes on tools on different manufacturing levels.

- Hard to predict tool availabilities in cause of variances in process stability.

- Optional process steps that enable for example effective measurement strategies.

- Time boundaries between process steps in cause of undesired chemical reactions.

The highly dynamic behaviour of wafer fabrication facilities states a big challenge in modeling the material flow at all. Especially foundries and manufacturer for logic products deal with dynamic circumstances like steady changes in equipment groups, product-mix and priorities in material flow control strategies. The enormous size and highly dynamic behaviour of wafer fabrication facilities makes an automated model generation of equipments and work flows desirable. But the high complexity makes a strategy to generate models automatically hard to implement . Based on reliable data, the automated generation of models promises high flexibility in modeling issues. Following that way models for simulator software could be created with much minor effort. 
Current IT-Systems in manufacturing grew over years to huge networks of interconnected databases. Therefore we intend to create one consistent database that holds all important information to reach our project goals. The process of collecting that data is characterized by the difficulty of identifying, extracting, and merging important information stored in different databases. Information quality plays a great role in that context. We focus on consistency, completeness, and accuracy of stored informations (Lee, Strong, Kahn, and Wang 2002). Verification and validation of extracted information is mainly done by hand. To ensure high degree of information quality for huge amounts of data we apply semi-automated comparisons when possible. For example we use such a automated cross checking comparison in the area of process times. We compare databases with planned process times and real historical process times stored in the manufacturing execution system (MES).

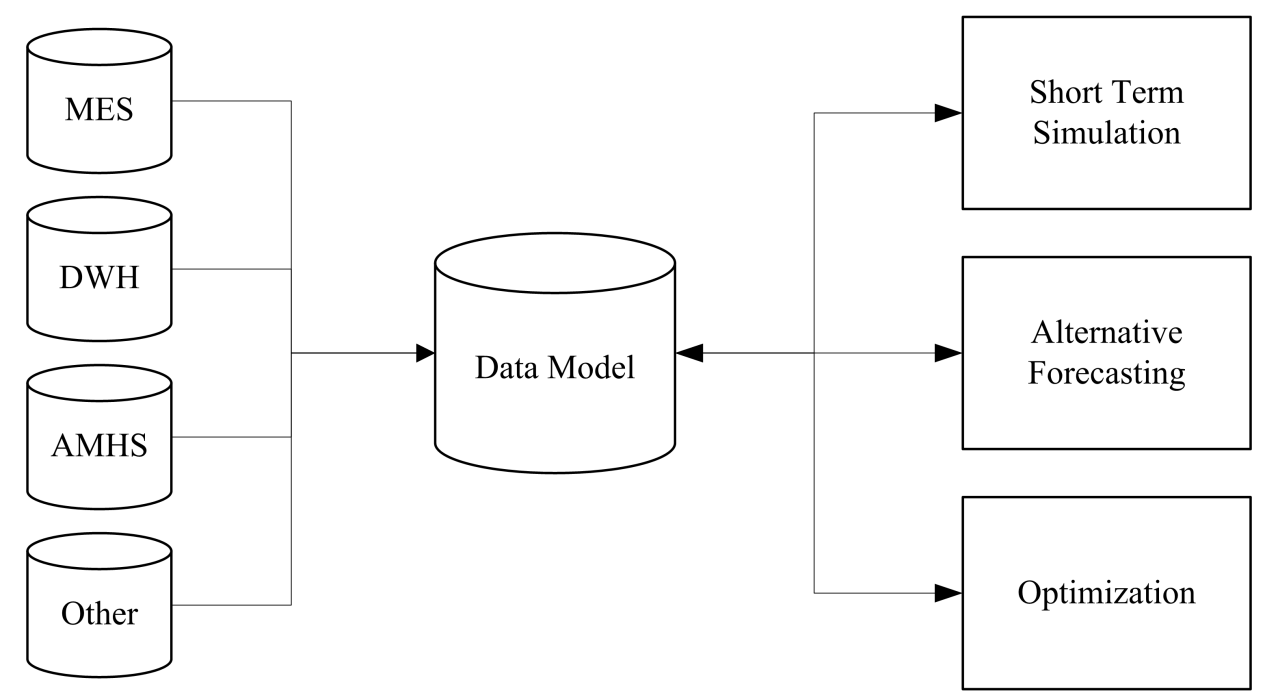

Figure 2: Architecture of our database

Our data model shown in Figure 2 is fed by several data sources, that are listed above:

- Manufacturing execution system (MES).

- Data ware house (DWH).

- Automated material handling system (AMHS).

- Other databases.

\section{SIMULATION ENABLES OPERATIONAL DECISION SUPPORT}

Simulation to generate reliable forecasts is state of the art in several induestries. Especially future performance prediction based on current waferfab status is desirable. The effectiveness of simulation methods strongly depends on the level of detail, the scale and the time horizon. Every forecast method highly depends on specified use cases. Forecast without decision making is almost useless. With respect to simulation based optimization, forecast is also an optimization of future performance. In the next two sections, we present the objectives of two approaches to predict future performance. We apply on the one hand discrete event simulation (DES) and on the other hand alternative forecasting.

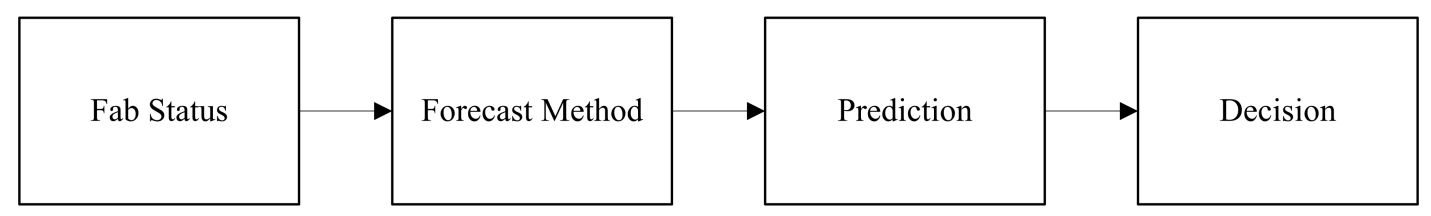

Figure 3: Future performance prediction enables decision support 


\subsection{Short Term Simulation Forecast}

For short term simulation we want to address multiple use cases on operational level. The use cases and methods define the scope of our model, the level of detail, and the forecast horizon. Matching the use cases and short term simulation setup is a bidirectional process. On the one hand multiple use cases have specific requirements in terms of the level of detail, the forecast horizon and the scale. On the other hand each method has its own capabilities. If the production department needs at least two weeks for a setup change but the method is capable to forecast only one week, then the forecasting method is not applicable to the setup change use case. We use an Oracle $\mathbb{R}$ database in combination with AutoSched $\mathrm{AP}^{\mathrm{TM}}$ as standard components for our implementation. For our short term simulation approach we create a full fab model to address the global scale use cases. The forecast time horizon is up to two weeks. It will be long enough to change dedications, setups and preventive maintenance schedules. The focus of the model is the transient period, and not the steady state (H.A. Reijers 1999). We want to reach high accuracy with a high precision initial state. The steady state is not our focus. In our forecast we predict critical performance indicators like work in progress (WIP), cycle time (CT) and lot arrivals per shift. The benefits of the forecast for our use cases depend on these future performance data. For one performance parameter, the trend over time is often more important than the mean value over time. To achieve our goal the following tasks are required:

- For the data collection we will take multiple data input sources into consideration. Our data model will query the current state of the fab and its master data. A historical data analysis will be applied for data which are not available directly (Skoogh and Johansson 2008). We gather data which are not available in an electronic format for example in expert interviews. These data have to be maintained manually in our database.

- For work center modeling, we have to address a desired accuracy, which highly depends on the available data. For each work center we have to break down the desired accuracy to work center features we want to integrate into our tool model. Features are setup, transportation, downs, additional resources, internal tool behaviour, e.g. like chamber models. We have to define representative tool models to model similar equipment types in the same manner. Equipments using the same model are different only in the input data, like processing time. An extended validation and verification work center is necessary. We are using historical arrival times to compare the model performance with historical tool performance in the reality system.

- The forecast gap analysis is important to increase the forecast accuracy and eliminate major errors (Bagchi, ChenRitzo, Shikalgar, and Toner 2008). We combine the work center models with the full fab model. The full fab simulation runs concurrently to reality. We compare the forecast results with real fab performance. The results of the gap analysis are major forecast errors and their reasons.

- The scenario manager initializes the fab with different model settings using the automated model generation. For this step the use cases have to be defined. Examples are PM planning or setup and dedication changes. The use cases highly depend on the level of detail in our model and our forecast time horizon. A GUI is necessary to import user settings like changed dedication.

- The automated optimization is an optional task. It will propose improved fab settings to reduce the effort of generating scenarios manually. An intelligent optimization heuristic is required to improve a specified objective by changing the fab settings.

All of these tasks are not independent. There may be several iterations of these tasks. For example if the forecast gap analysis shows that the cluster tool model does not have the proper accuracy for forecasting, a more detailed cluster tool model is required. Expected forecast error reasons are sampling, process exceptions, variances in process and transport durations. Some characteristics are summarized in Table 1.

Table 1: Short term simulation characteristics

\begin{tabular}{l|l} 
Characteristic & Description \\
\hline Use cases & Forecasting as decision support for PM, dedication, setup, and standby planning \\
Level of detail & WIP, CT, and lot arrivals per shift, for all operations/work centers \\
Scale & Full fab without minor equipment \\
Computation time & A few minutes to simulate two weeks of a wafer fab
\end{tabular}




\subsection{Alternative Forecasting}

In contrast to short term simulation forecast the alternative forecasting does not need the full fab model information. The whole purpose of alternative prediction is to simplify the solution by heuristic methods. The basis for the approach is a statistical evaluation of historical data. We analyze dependencies between the output characteristics and a variety of output parameters (Armstrong 2001). A detailed requirement specification is important, because of the fact that the alternative forecasting methods are focused on the problem they should solve (Box and Jenkins 1994). Our objective is to make a forecast of job arrival trends for several work center in the waferfab.

The forecast uses the same database as the short time simulation, but it needs some different information with precomputed values for time intervals between a start and a target operation (Duan and Babu 2007). In reality the start operation is not the first operation in the route. It is the current lot position in the route before a selected target operation as shown in Figure 4.

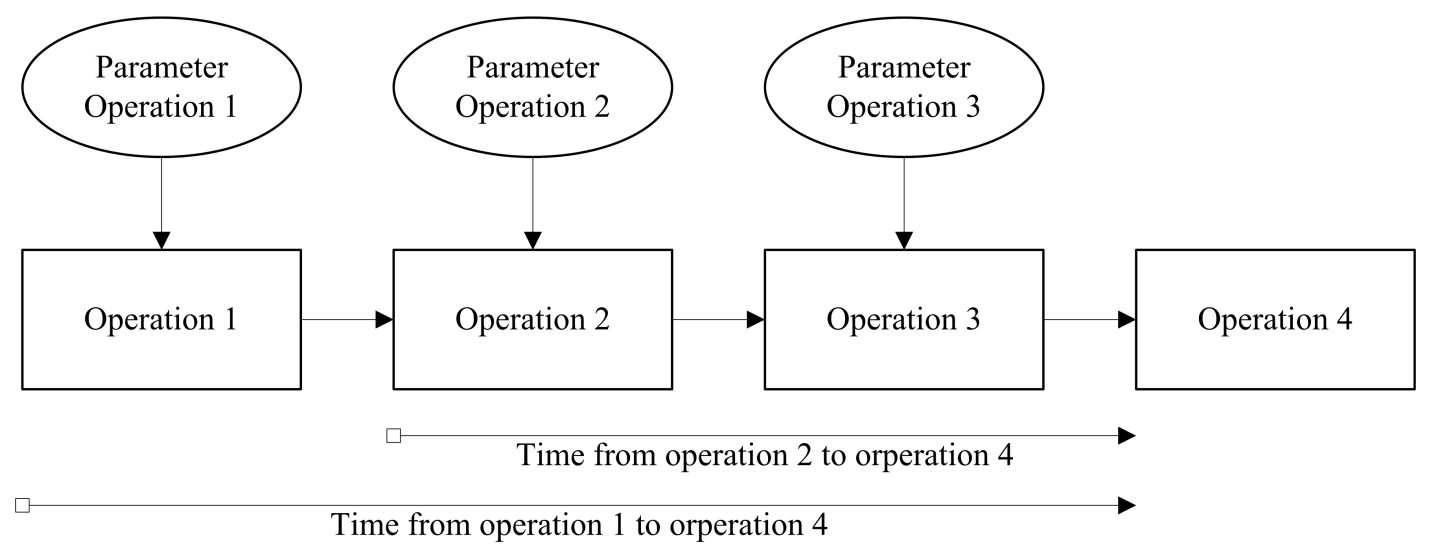

Figure 4: Methodology of alternative forecasting

It is fundamental that for the same product the duration between the operations depends on other factors, too. It is necessary to cluster historical data dependent on parameters like priority, product type, number of wafer or others. The result of this data preparation is a collection of time intervals between process steps. They provide time information from every predecessor operation to a target operation.

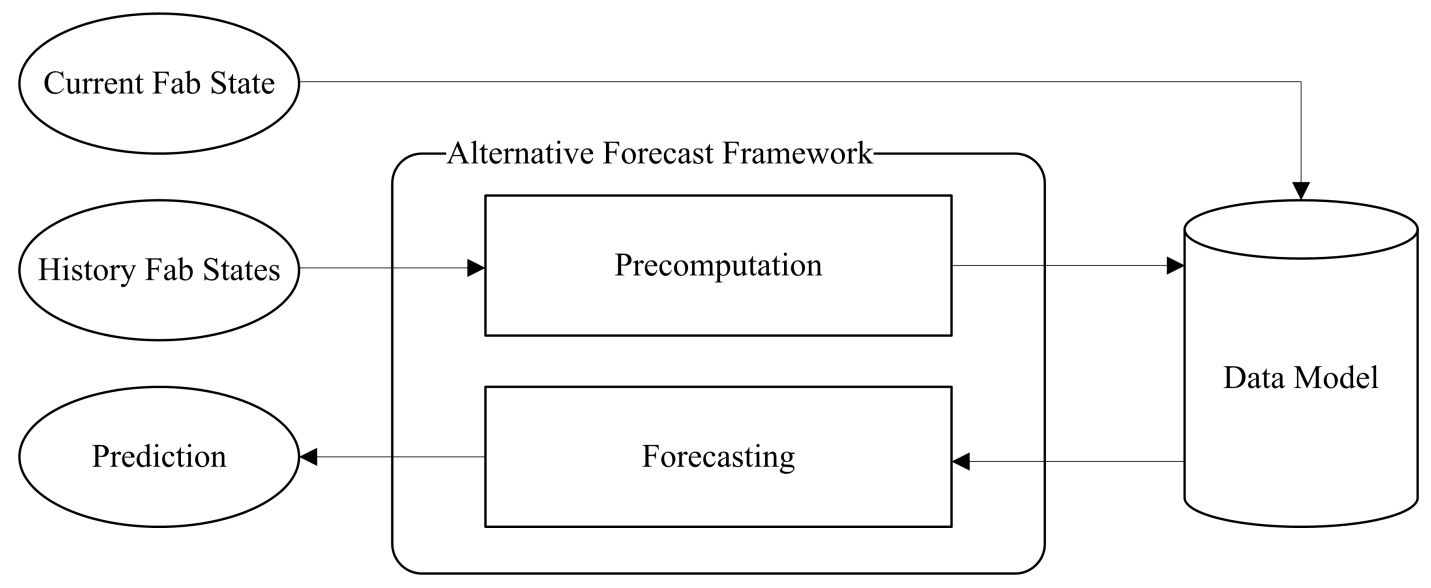

Figure 5: Data flow of alternative forecasting concept

The alternative forecasting method under development works in two steps. In the first step the forecasting method extracts information like the current waferfab WIP. For each lot the current state is available. The second step involves the 
computation of the already calculated duration between the current and the target operation for every lot. To compute this duration, the historical data have to be updated in small time periods (daily) to reproduce current waferfab behavior. Older data receive a smaller weight as actual values, but they are still important to smooth the parameters that describe a factory state.

In industry the alternative forecasting is an important alternative to simulation (Powers, Goldszmidt, and Cohen 2005). We try to show that the solution of many problems does not need a complex simulation solution. The reason is that simulation models need detailed equipment information which include a lot of effort. In our project we have the possibility to make a comparison of both forecasting methods. The main characteristics are shown in Table 2.

Table 2: Alternative forecasting characteristics

\begin{tabular}{l|l} 
Characteristic & Description \\
\hline Use cases & Future performance prediction \\
Level of detail & WIP, CT, Lot arrivals per shift, per operation \\
Scale & Single operations, applicable for all operations in the fab. \\
Computation time & A few seconds to compute the prediction for one operation
\end{tabular}

\section{OPTIMIZATION}

Current rule based dispatching on operational level is state of the art in the semiconductor industry. These dispatching systems control the material flow without using optimization techniques. The challenge is to achieve global strategic goals under consideration of local operational needs.

On one hand several manufacturers extend their dispatching systems with simulation capabilities (Kalisch, Ringel, and Weigang 2008). In combination with optimizing approaches this extension leads to more effective work flow management systems. Almost always simulation based optimization approaches were based on established rule based dispatching systems. There is an obvious tendency to multi layered control systems that combine simulation and dispatching mechanisms.

On the other hand solver based optimization or the use of heuristics to solve optimization problems becomes popular. These approaches usually focus on specified subproblems due to high computational complexity. Currently available optimization techniques are able to solve most subproblems with sufficient accuracy in acceptable time.

Most manufacturers try to establish optimized resource scheduling on operative level. The vision is an industrial implementation of operative real time scheduling on lot level in waferfabs. In fact scheduling on operative level is very hard to achieve because of high complexity.

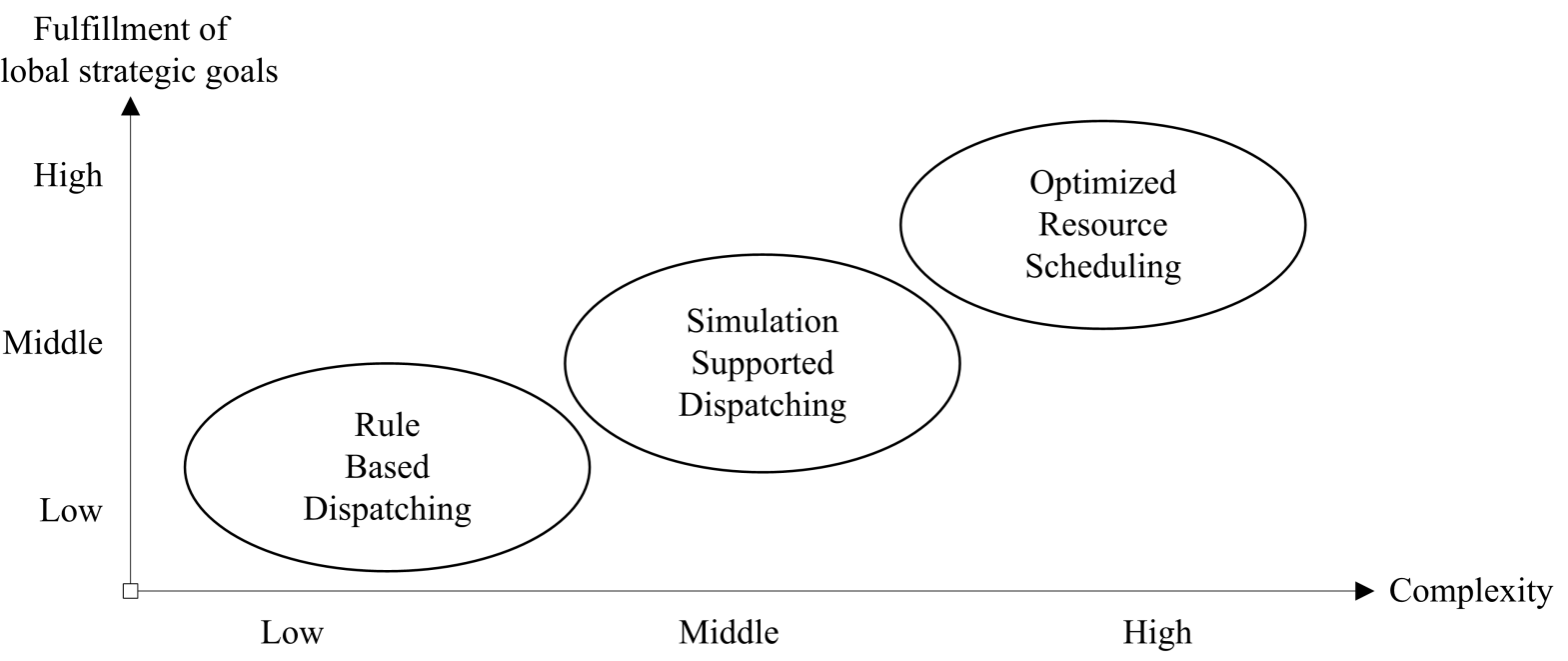

Figure 6: Overview about WFM methods 
Several effects lead to high complexity and highly dynamic behaviour of waferfabs. These challenges have to be considered by optimization approaches. Some of these are listed below:

- Non-predictable events like equipment breakdown.

- Permanent changes in equipment groups.

- Changing product-mix.

- Changing priorities in material flow control strategies

- High variance in process and transport times.

To handle computational complexity it is necessary to split optimization problems into subproblems. For instance we group equipments to work centers to define solvable optimization problems. Like scheduling lots on tools in a specified time frame in order to improve a given objective function.

The potential benefit in combination with the degree of complexity determines the usability of optimizing approaches. That relation determines the acceptance of these approaches in practice. We will evaluate different approaches in our project.

- Simulation based optimization.

- Solver based optimization.

- Optimization using search methods.

- Artificial intelligence techniques.

One of our first steps was to optimize batch processes of furnaces. We were able to show the usability of solver based optimization techniques on furnace batch processes (Klemmt, Hielscher, Horn, and Weigert 2008). Cycle time improvements can be achieved under consideration of all real constraints in fabrication environment.

In addition we expect synergy effects in our project concept. Beyond the usage of the same database sharing results between work packages would be positive. E.g. we plan to feed optimization approaches with lot arrival events derived from simulation.

\section{CONCLUSION \& OUTLOOK}

This paper presents the goals, methods and challenges of our project in the application field of modeling, simulation and optimization of work flows in semiconductor industry. Our goal is to evaluate suitable methods in above mentioned application areas in use cases given by reality. This project considers future performance prediction using different simulation techniques. In addition several optimization approaches will be evaluated in this project.

Analysis of systems and constraints is already done. But more detailed studies on selected topics are necessary to understand existing relationships in detail. The development of an data infrastructure that feeds our data model is in progress. First models were created. Based on these first results we evaluated solver based optimization approach in the field of furnace batch optimization. In the application area of simulation first forecasts approaches are ready to be used.

At the moment we deal with several challenges to increase the granularity of our models. For example measurement issues and related sampling strategies seem to be very complex and hard to model. Improving quality and extension of our models will be the following steps in our project.

\section{REFERENCES}

Armstrong, J. E. 2001. Principles of forecasting a handbook for researchers and practitioners, Volume 30 of International Series in Operations Research \& Management Science. Springer.

Bagchi, S., C.-H. Chen-Ritzo, S. T. Shikalgar, and M. Toner. 2008. A full-factory simulator as a daily decision-support tool for 300mm wafer fabrication productivity. In Proceedings of the 2008 Winter Simulation Conference, eds. S. J. Mason, R. R. Hill, L. Mönch, O. Rose, T. Jefferson, J. W. Fowler, 2021-2029: Piscataway, New Jersey: Institute of Electrical and Electronics Engineers, Inc.

Box, G. E. P., and G. M. Jenkins. 1994. Time series analysis: Forecasting and control. Upper Saddle River, NJ, USA: Prentice Hall PTR.

Duan, S., and S. Babu. 2007. Processing forecasting queries. In Proceedings of the 33rd international conference on Very large data bases (VLDB '07), 711-722: VLDB Endowment. 
H.A. Reijers, W. v. d. A. 1999. Short-term simulation: Bridging the gap between operational control and strategic decision making. In Proceedings of the 1999 IASTED International Conference on Modeling and Simulation.

Kalisch, S., R. Ringel, and J. Weigang. 2008. Managing wip and cycle time with the help of loop control. In Proceedings of the 2008 Winter Simulation Conference, eds. S. J. Mason, R. R. Hill, L. Mönch, O. Rose, T. Jefferson, J. W. Fowler, 2298-2304: Piscataway, New Jersey: Institute of Electrical and Electronics Engineers, Inc.

Klemmt, A., T. Hielscher, S. Horn, and G. Weigert. 2008. Simulations-based and solver-based optimization approaches for batch processes in semiconductor manufacturing. In Proceedings of the 2008 Winter Simulation Conference, eds. S. J. Mason, R. R. Hill, L. Mönch, O. Rose, T. Jefferson, J. W. Fowler, 2041-2049: Piscataway, New Jersey: Institute of Electrical and Electronics Engineers, Inc.

Lee, Y. W., D. M. Strong, B. K. Kahn, and R. Y. Wang. 2002. AIMQ: A methodology for information quality assessment. Information \& Management 40 (2): 133 - 146.

Powers, R., M. Goldszmidt, and I. Cohen. 2005. Short term performance forecasting in enterprise systems. In Proceedings of the 11th ACM SIGKDD International Conference on Knowledge Discovery in Data Mining, 801-807. New York, NY, USA: ACM.

Schönherr, O. 2008. Ein allgemeines SysML Modell zur Abbildung diskreter Prozesse in der Produktion. Master's thesis, Technische Universität Dresden.

Skoogh, A., and B. Johansson. 2008. A methodology for input data management in discrete event simulation projects. In Proceedings of the 2008 Winter Simulation Conference, eds. S. J. Mason, R. R. Hill, L. Mönch, O. Rose, T. Jefferson, J. W. Fowler, 1727-1735: Piscataway, New Jersey: Institute of Electrical and Electronics Engineers, Inc.

\section{AUTHOR BIOGRAPHIES}

ROBERT KOHN is a PhD student at Dresden University of Technology. His focus is on modeling and optimization of material flows, especially in semiconductor industry. He is a member of the scientific staff of Prof. Dr. Oliver Rose at the Chair of Modeling and Simulation. He received his M.S. degree in computer science from University of Applied Sciences Stralsund. His email address is <robert.kohnetu-dresden. de>.

MARCIN MOSINSKI is a PhD student at Dresden University of Technology. He is a member of the scientific staff of Prof. Dr. Oliver Rose at the Chair of Modeling and Simulation. He received his M.S. degree in computer science from Dresden University of Technology. His research interests include forecasting of complex problems in manufacturing facilities and the statistical data analysis. His email address is <marcin.mosinski@tu-dresden. de>.

DANIEL NOACK is a Research Engineer at D-SIMLAB Technologies. His focus is on simulation and simulation-based optimization. He is also a PhD student at Dresden University of Technology. He is a member of the scientific staff of Prof. Dr. Oliver Rose at the Chair of Modeling and Simulation. He received his M.S. degree in computer science from Dresden University of Technology. His email address is <daniel.noackeinf.tu-dresden.de>.

ZHUGEN ZHOU is a PhD student at Dresden University of Technology. He is a member of the scientific staff of Prof. Dr. Oliver Rose at the Chair of Modeling and Simulation. He received his M.S. degree in Computational Engineering from Dresden University of Technology. His research interests include dispatching concepts for complex production facilities and work center modeling for wafer fab. His email address is <zhugen.zhou@tu-dresden.de>.

OLIVER ROSE holds the Chair for Modeling and Simulation at the Institute of Applied Computer Science of the Dresden University of Technology, Germany. He received an M.S. degree in applied mathematics and a Ph.D. degree in computer science from Würzburg University, Germany. His research focuses on the operational modeling, analysis and material flow control of complex manufacturing facilities, in particular, semiconductor factories. He is a member of IEEE, INFORMS Simulation Society, ASIM, and GI. He will be the General Chair of WSC 2012 at Berlin. Web address: <www. simulation-dresden. com>. 